\title{
Inertial currents in isotropic plasma
}

\section{Heinemann}

Phillips Laboratory, Hanscom Air Force Base, Massachusetts

G. M. Erickson

Center for Space Physics, Boston University, Boston, Massachusetts

D. H. Pontius Jr.

Bartol Research Institute, Newark, Delaware

\begin{abstract}
The magnetospheric convection electric field contributes to Birkeland currents. The effects of the field are to polarize the plasma by displacing the bounce paths of the ions from those of electrons, to redistribute the pressure so that it is not constant along magnetic field lines, and to enhance the pressure gradient by the gradient of the bulk speed. Changes in the polarization charge during the convection of the plasma are neutralized by electrons in the form of field-aligned currents that close through the ionosphere. The pressure drives field-aligned currents through its gradient in the same manner as in quasi-static plasma, but with modifications that are important if the bulk speed is of the order of the ion thermal speed; the variations in the pressure along field lines are maintained by a weak parallel potential drop. These effects are described in terms of the field-aligned currents in steady state, isotropic, MHD plasma. Solutions are developed by taking the MHD limit of two-fluid solutions and illustrated in the special case of Maxwellian plasma for which the temperature is constant along magnetic field lines. The expression for the Birkeland current density is a generalization of Vasyliunas' expression for the field-aligned current density in quasi-static plasma and provides a unifying expression when both pressure gradients and ion inertia operate simultaneously as sources of field-aligned currents. It contains a full account of different aspects of the ion flow (parallel and perpendicular velocity and vorticity) that contribute to the currents. Contributions of ion inertia to field-aligned currents will occur in regions of strong velocity shear, electric field reversal, or large gradients in the parallel velocity or number density, and may be important in the low-latitude boundary layer, plasma sheet boundary layer, and the inner edge region of the plasma sheet.
\end{abstract}

\section{Introduction}

Birkeland currents in the Earth's magnetosphere constitute the mechanism by which the magnetosphere is coupled to the ionosphere. The drifts of charged particles, electrons and ions, in the magnetosphere carry current perpendicular to the magnetic field lines. The perpendicular currents generally cannot close in the magnetosphere. The requirement that the currents must

\section{Copyright 1994 by the American Geophysical Union.}

Paper number 94JA00204.

0148-0227/94/94JA-00204\$05.00 
close on an Alfvén transit timescale in order to maintain quasi-neutrality means that they must close in the ionosphere via Birkeland currents. The closure currents determine the electric field in the resistive ionosphere, which in turn determines the electric field in the magnetosphere, modifying the magnetospheric drifts. The behavior and evolution of the system is thus determined by the Birkeland currents. The convection electric field is always present and plays a primary role in the determination of the currents. Any description of the origin of Birkeland currents should in principle take the convection electric field into account.

In the context of fluid or MHD equations the effects of the convection electric field are described in terms of ion inertia, the time rate of change of the ion mean velocity, or equivalently, in terms of ion vorticity, velocity shear, or velocity gradients. It is known [Hasegawa and Sato, 1979; Ogino, 1986; Southwood and Kivelson, 1991; Birn and Hesse, 1991] that ion vorticity contributes to fieldaligned currents. The importance of the effects of ion inertia in magnetospheric plasma can be surmised from flow of plasma within different regions of the magnetosphere. The association of field-aligned currents [Coley, 1983], auroras [Chiu and Gorney, 1983], and the boundary plasma sheet [Lyons, 1981] with convection reversals suggest an intrinsic relation between field-aligned currents and magnetospheric flows. In the plasma-sheet boundary layer, flow velocities of the order of hundreds of kilometers per second in a layer about an Earth radius thick are known to persist for hours [Hones et al, 1976; Eastman et al, 1984, 1985]. The low-latitude boundary layer [Eastman et al., 1976, Eastman and Hones, 1979] shows velocity gradients that result from the braking of the magnetosheath flow, of the order of $1000 \mathrm{~km} \mathrm{~s}^{-1}$ per Earth radius, and can be expected to contribute to the field-aligned currents as a result of the gradients in addition to viscous transport [Sonnerup, 1980; Phan et al., 1989]. Flows near the inner edge of the plasma sheet, which must turn sharply near the shielding layer, can also be expected to show contributions resulting from the ion acceleration. McFadden et al. [1986] observe that convection of cold plasma above the field-aligned acceleration region can account for their observations of precipitation at the edge of an arc. Galperin and Volosevich [1989] have shown that the polarization drift affects field-aligned currents if the electric field varies on the scale of the ion gyroradius and argue that it plays a role in the formation of narrow, elongated, auroral arcs.

It has long been argued that the theory of fieldaligned currents is inadequate because existing models treat only one generator (pressure gradients, ion inertia, or viscosity) at a time [Akasofu, 1984] (see also Siscoe and Fedder [1993]). For example, the theories of 
Vasyliunas [1970], Rostoker and Boström [1976], and Sonnerup [1980] are restricted to pressure gradients, ion inertia, and viscosity, respectively, as the source of the field-aligned currents. The development of a theory that simultaneously treats all three is an important matter for geospace global circulation models that attempt to combine the different regions of the magnetosphere (magnetosheath, magnetopause boundary layer, magnetosphere, and ionosphere) into a single, coupled, unified model. The purpose of this paper is to develop the theory of one level of unification of the generators - the simultaneous treatment of pressure gradients and ion inertia.- Specifically, we propose to quantify the relations among the field-aligned current density at the ionosphere, that is, the Birkeland current density, and the convection electric field and pressure gradients in steady state, isotropic, MHD plasma. Starting with a two-fluid description of the plasma, we derive an expression for the current density that explicitly accounts for convection. The expression is a straightforward generalization of those of Vasyliunas [1970] and Tverskoy [1982] to include the effects of electric and gravitational fields. The expression for the field-aligned current density assumes that equilibrium is established along the magnetic field and is therefore valid on a timescale long compared to the particle bounce time and the Alfvén transit time, the time it takes for an Alfvén wave to travel the length of a field line. The results can be applied to long-lasting Birkeland currents, but not rapidly varying systems such as substorm currents. Both re strictions can be removed, but only at the expense of algebraic complications.

The phenomenological basis for the assumption of isotropic pressure is the observation that magnetospheric plasma is often isotropic to a good approximation [e.g., Stiles et al., 1978]. At the same time, the plasma is also collisionless, at least in the sense of Coulomb collisions. We note that purely collisionless drifting plasma generally cannot remain isotropic because the drifts energize the parallel and perpendicular motions of the particles at different rates (see Hines, 1963). The essential assumption of our analysis is that the bounce motions can be described by the behavior of collisionless isotropic plasma but that isotropy is maintained by some mechanism, not explicitly treated, on a drift timescale; such mechanisms include fluid (firehose and mirror) instabilities [Nötzel et al, 1985], chaos in the plasma sheet [Büchner and Zelenyi, 1989], and strong pitch angle scattering [Kennel and Petschek, 1966]. The manner in which we take this assumption into account is to assume that the equatorial distribution functions are isotropic but that the bounce motions are collisionless. The assumption of isotropy can easily be replaced by one of gyrotropy using the methods of Heinemann 
and Pontius [1991] (hereafter called HP91). To include gyrotropic pressure in the present paper would merely serve to complicate the presentation of what turns out to be a relatively simple physical picture. The mathematical details of time dependence and gyrotropic pressure will be published elsewhere.

The principal formal result of the paper is the generalization of Vasyliznas' [1970] formula for the fieldaligned current density in quasistatic plasma to include the effects of ion inertia, gravity, and parallel potential drops; the reader is referred to equation (69) and those that follow. The expression, which is based on the as sumption of isotropic Maxwellian distribution functions in steady state, is somewhat more complicated than that for quasi-static plasma but should be useful for the computation of Birkeland currents. The principal physical results of the paper are that the convection electric field has three main effects on Birkeland currents: it polarizes the plasma by displacing the bounce motions of the ions from those of electrons, it adjusts the pressure to satisfy equilibrium along the magnetic field, and it enhances the pressure gradient by the gradient of the bulk energy. The polarization causes a charge separation of ions and electrons that convects at the $E \times B$ drift velocity. Neutralization of the excess charge by electrons is accomplished by currents that close through the ionosphere, that is, Birkeland currents. The parallel pressure equilibrium is maintained by a weak parallel electric field that survives in MHD approximation. The pressure gradients contribute to the Birkeland currents through their gradients in the same manner as in quasi-static plasma, but with modifications that are important if the ion velocity is of the order of the ion thermal speed. In addition, the gradient of the square of the ion velocity contributes, essentially by adding to the pressure gradient.

The paper is divided into three sections. In the first section we develop expressions for field-aligned currents in the terms of a two-fluid description of the plasma, assuming that the ion and electron pressures are isotropic and the system is in steady state. In the second section we take the MHD limit of the two-fluid solutions and illustrate the results in terms of isotropic Maxwellian distribution functions for which the temperature along the magnetic field is constant. We conclude with a discussion of the main results.

\section{Two-Fluid Analysis}

From the perspective of fluid behavior of magnetospheric plasma the origin of the field-aligned currents is the plasma momentum. Gradient-curvature drifts, the ion inertial drift, and the gravitational drift all con- 
tribute to the momentum density of the plasma, and must be balanced by the $J \times B$ force in steady state. This balance is expressed mathematically by the momentum equation for quasi-neutral plasma

$$
\rho \frac{d \mathbf{V}}{d t}+\operatorname{div} \mathcal{P}+\rho \nabla \phi=\frac{1}{c} \mathbf{J} \times \mathbf{B},
$$

where $\rho$ is the mass density, $\mathbf{V}$ is the bulk velocity, $\mathcal{P}$ is the kinetic tensor in the proper frame of the plasma, $\phi$ is the gravitational potential, B is the magnetic field, $\mathbf{J}$ is the electric current density, and $c$ is the speed of light; units are cgs. The tensor $\mathcal{P}$ is defined by

$$
\mathcal{P}_{i j}=\sum_{a}\left[P_{a} \delta_{i j}+m_{a} n_{a}\left(V_{a i}-V_{i}\right)\left(V_{a j}-V_{j}\right)\right],
$$

where $a$ denotes the species of particle (electrons and ions), $m_{a}$ is the mass of the species, $n_{a}$ is its number density, $P_{a}$ is its scalar pressure, and $V_{a}$ is its mean velocity [see Rossi and Olbert, 1970, p. 290]. The convective derivative is $d / d t=\partial / \partial t+V \cdot \nabla$ and reduces to $V \cdot \nabla$ in steady state.

Equation (1) determines the component of the current density perpendicular to the magnetic field in terms of the other quantities, that is, in terms of the perpendicular drifts. By itself, it is insufficient to determine the total current density. The parallel current density must be determined by the requirement that the total current density be solenoidal on an Alfvén transit time scale,

$$
\nabla \cdot \mathbf{J}=0,
$$

in order to prevent rapid charging of the plasma [see Vasyliunas, 1970].

The solution of (1)-(3) for the field-aligned current density has been approached in MHD approximation by a number of authors. The difficulty in obtaining solutions is the behavior of the inertial current

$$
\mathrm{J}_{P} \equiv \frac{\rho c}{B^{2}} \mathbf{B} \times \frac{d \mathbf{V}}{d t},
$$

which does not easily lend itself to mathematical analysis. Rather than work in terms of MHD plasma, a natural way to include inertial effects is to follow Hasegawa and Sato [1979] by writing separate equations governing mass and momentum of the electrons and ions. A benefit of working with separate fluid equations is that they lead to analogs of the one-fluid equations in which the equilibrium plasma is described by a set of field line constants and which can be solved in much the same way as in quasi-static plasma. The resulting two-fluid solution can be reduced by appropriate approximations to the MHD limit. 
The laws governing the two-fluid motions are mass conservation,

$$
\frac{\partial n_{a}}{\partial t}+\nabla \cdot n_{a} V_{a}=0
$$

and the momentum equation,

$m_{a} n_{a} \frac{d_{a} V_{a}}{d t}+\nabla P_{a}+m_{a} n_{a} \nabla \phi=n_{a} q_{a} \mathrm{E}+\frac{n_{a} q_{a}}{c} V_{a} \times \mathrm{B}$

where $\mathbf{E}$ is the electric field. Equation (5) is valid for each species of particle (electrons and ions) as long as there are no sources or sinks of each species, for example, ionization, recombination, or charge exchange. Equation (6) is valid for plasma in which the momentum exchange between species can be neglected and in which the pressure can be regarded as isotropic. Anisotropic pressure and viscosity have been neglected; they can be taken into account but only at the expense of algebraic complications. The assumption of isotropic pressure neglects the so-called finite Larmor radius effects, which are important in collisionless plasma on the scale of an ion gyroradius [Macmahon, 1965; Stasiewicz, 1989, 1991]. The convective derivative, $d_{a} / d t \equiv \partial / \partial t+V_{a} \cdot \nabla$, depends on the species.

To develop (6) in a form amenable to analytic treatment, we express the electric and magnetic fields in terms of potentials, $\mathbf{E}=-\operatorname{grad} \Phi-(1 / c) \partial \mathbf{A} / \partial t$ and $\mathbf{B}=\operatorname{curl} \mathbf{A}$, where $\Phi$ is the electrostatic potential and $\mathbf{A}$ is the vector potential. Then the momentum equation can be written

$$
\begin{aligned}
\frac{\partial \Pi_{a}}{\partial t} & -\frac{q_{a}}{c} V_{a} \times \Gamma_{a} \\
& =-\frac{1}{n_{a}} \nabla P_{a}-\nabla\left(q_{a} \Phi+m_{a} \phi+\frac{1}{2} m_{a} V_{a}^{2}\right),
\end{aligned}
$$

where $\Pi_{a}=m_{a} V_{a}+\left(q_{a} / c\right) A$ is the "canonical momentum" associated with the fluid velocity, and the "generalized vorticity"

$$
\Gamma_{a} \equiv \frac{c}{q_{a}} \nabla \times \Pi_{a}=\mathrm{B}+\frac{m_{a} c}{q_{a}} \omega_{a}
$$

is the curl of the canonical momentum, and $\omega_{a} \equiv$ curl $V_{a}$ is the ordinary fluid vorticity; the units have been chosen such that $\Gamma_{a}$ has the same dimensions as B.

We seek solutions in which equilibrium is established along the magnetic field, and therefore neglect the partial time derivative. In this case the velocity perpendicular to $\Gamma_{a}$ is

$$
\begin{aligned}
\mathbf{V}_{a \perp}= & \frac{c}{q_{a} \Gamma_{a}^{2}} \Gamma_{a} \times\left[\frac{1}{n_{a}} \nabla P_{a}\right. \\
& \left.+\nabla\left(q_{a} \Phi+m_{a} \phi+\frac{1}{2} m_{a} V_{a}^{2}\right)\right]
\end{aligned}
$$


and the mass conservation equation (5) reduces to

$$
\nabla \cdot n_{a} V_{a}=0
$$

It follows from (7) that

$$
\Gamma_{a} \cdot\left[\frac{1}{n_{a}} \nabla P_{a}+\nabla\left(q_{a} \Phi+m_{a} \phi+\frac{1}{2} m_{a} V_{a}^{2}\right)\right]=0 .
$$

Equations (9)-(11) are two-fluid analogs of the quasistatic isotropic one-fluid equations $\mathrm{J}_{\perp}=\left(c / B^{2}\right) \mathbf{B} \times \nabla P$, $\operatorname{div} \mathbf{J}=0$, and $\mathbf{B} \cdot \nabla P=0$ that lead in quasi-static plasma to expressions for the parallel current density in terms of gradients of the flux-tube volume and pressure gradients [Vasyliunas, 1970; Heinemann and Pontius, 1990 (hereafter called HP90)]. Like $B, \Gamma_{a}$ is divergencefree; one may therefore associate flux tubes with $\boldsymbol{\Gamma}_{a}$ analogous to magnetic flux tubes. By exploiting the analogies, one can include the effects of ion inertia.

A solution of (11) can be obtained by writing the number density and pressure in the forms

$$
\begin{gathered}
n_{a}(\mathbf{x})=\frac{2^{3 / 2} \pi}{m_{a}^{3 / 2}} \int \sqrt{\mathcal{E}-q_{a} \Phi-m_{a} \phi-\frac{1}{2} m_{a} V_{a}^{2}} \\
\cdot f_{a}\left(\mathcal{E} ; C_{j}\right) d \mathcal{E} \\
P_{a}(\mathbf{x})=\frac{2^{5 / 2} \pi}{3 m_{a}^{3 / 2}} \int\left(\mathcal{E}-q_{a} \Phi-m_{a} \phi-\frac{1}{2} m_{a} V_{a}^{2}\right)^{3 / 2} \\
\cdot f_{a}\left(\mathcal{E} ; C_{j}\right) d \mathcal{E},
\end{gathered}
$$

where $\mathcal{E}$ represents particle total energy and $f_{a}\left(\mathcal{E}, C_{j}\right)$ is the particle distribution function expressed as a function of particle total energy and arbitrary constants, $C_{j}$, that are used to specify the equatorial distribution function. These are appropriate for plasma that is isotropic in the proper frame of each species (i.e., the frame in which the velocity of each species vanishes). One can verify by direct substitution that these solve (11) if it is assumed that $\Gamma_{a} \cdot \nabla C_{j}=0$.

The condition $\Gamma_{a} \cdot \nabla C_{j}=0$ is related to the polarization of the plasma by the convection electric field. Its physical significance can be sought in terms of a Vlasov description of the bounce motions of the particles. From the guiding-center theory of plasma motions [e.g., Northrop, 1963] it can be shown that, if the $E \times B$ drift velocity has a curl, then the guiding center velocity is given by

$$
\mathbf{v}_{a}=\frac{v_{a \| l}}{B}\left[\mathrm{~B}+\frac{m_{a} c}{q}\left(\nabla \times \mathrm{V}_{E}\right)_{\perp}\right]+\cdots
$$

where $\mathrm{V}_{E}=c \mathrm{E} \times \mathrm{B} / B^{2}$ represents the $E \times B$ drift and the ellipsis includes the $E \times B$, gradient, curvature, and $g \times B$ drifts. The second term, which is equivalent to the polarization drift, means that particles are 
displaced in their bounce motions by a distance that depends on the perpendicular vorticity. At an intuitive level this means that distribution function is constant along field lines defined by $\mathrm{B}+\left(m_{a} c / q_{a}\right)\left(\text { curl } V_{E}\right)_{\perp}$ as are the constants characterizing the distribution function. A tedious calculation (not given) shows that finite gyroradius corrections lead to $\Gamma_{a} \cdot \nabla C_{j}=\mathcal{O}\left[\left(m_{a} c / q_{a}\right)^{2}\right]$, where $\Gamma_{a}$ is based on the mean velocity of the species including the "magnetization" contribution to velocity, $\mathrm{V}_{a}^{(M)}=-\left(c / n_{a} q_{a}\right) \operatorname{curl}\left(P_{\mathrm{a}} \mathrm{B} / B^{2}\right)$. At this order of an expansion in powers of $m_{a} c / q_{a}$ this result does not distinguish between the $E \times B$-drift and the perpendicular component of the mean velocity of the species. It does, however, indicate the physical basis of the solution of (11) by (12) and (13) lies in the polarization displacements of the particles in their bounce motions. Moreover, since it will ultimately be used only in the MHD limit, which also does not distinguish between $\mathbf{V}_{E}$ and $V_{a \perp}$, it is entirely adequate for our purposes to assume that $\Gamma_{a} \cdot \nabla C_{j}=0$.

As an example of (12) and (13), consider a convected Maxwellian particle distribution

$$
f_{a, e}(\mathbf{v})=\frac{n_{a, e}}{\left(2 \pi T_{a, e} / m_{a}\right)^{3 / 2}} e^{-m_{a}\left(v-V_{a, e}\right)^{2} / 2 T_{a, e}},
$$

where $n_{a, e}$ is the equatorial number density, $T_{a, e}$ is the equatorial temperature in ergs, and $\mathbf{V}_{a, e}$ is the equatorial velocity. Because of the assumed isotropy in the moving frame, this can be expressed in terms of the total energy and equatorial constants:

$$
\begin{gathered}
f_{a}\left(\mathcal{E} ; C_{j}\right)=\frac{n_{a, e}}{\left(2 \pi T_{a, e} / m_{a}\right)^{3 / 2}} \\
\cdot \exp \left[-\frac{\mathcal{E}-q_{a} \Phi_{e}-m_{a} \phi_{e}-\frac{1}{2} m_{a} V_{a, e}^{2}}{T_{a, e}}\right]
\end{gathered}
$$

The number density and pressure that follow from integration of (12) and (13) are

$$
\begin{aligned}
& n_{a}=n_{a, e} e^{-q_{a}\left(\Psi_{a}-\Psi_{a, e}\right) / T_{a, e}} \\
& P_{a}=P_{a, e} e^{-q_{a}\left(\Psi_{a}-\Psi_{a, e}\right) / T_{a, e}},
\end{aligned}
$$

where $\Psi_{a}$ represents the "effective potential" defined by

$$
q_{a} \Psi_{a} \equiv q_{a} \Phi+m_{a} \phi+\frac{1}{2} m_{a} V_{a}^{2} .
$$

(In writing (17) and (18), the effects of the loss cone have been neglected so that the pressure can be assumed to be isotropic all the way to the ionosphere. While this assumption is incorrect in application to magnetospheric plasma, the magnitude of the error involved is 
small, a few percent; see HP91 for an explicit evaluation in quasi-static plasma.) The number density and pressure are explicit functions of the equatorial constants $n_{a, e}, \Phi_{e}, \phi_{e}, V_{a, e}$, and $T_{a, e}$. The meaning of (17) and (18) is that they are the number density and pressure at a point that is connected by field lines of $\Gamma_{a}$ to the equatorial crossing point with values $n_{a, e}, \Phi_{e}, \phi_{e}, V_{a, e}$, and $T_{a, e}$.

Equations (12) and (13) solve the parallel equilibrium problem and thereby determine the three-dimensional distribution of $n_{a}$ and $P_{a}$ for given potentials and equatorial distribution functions. The distributions of $n_{a}$ and $P_{a}$ in turn determine the plasma drift velocities. Differentiation of (13) and use of (12) (or (18) and (17)) to identify $n_{a}$ shows that the following differential relations hold: $\partial P_{a} / \partial \Phi=-q_{a} n_{a}, \partial P_{a} / \partial \phi=-m_{a} n_{a}$, and $\partial P_{a} / \partial \frac{1}{2} V_{a}^{2}=-m_{a} n_{a}$. These differential relations show that the gradient of the pressure, obtained by taking the gradient of (13), is

$$
\begin{aligned}
\nabla P_{a} & =\frac{\partial P_{a}}{\partial \Phi} \nabla \Phi+\frac{\partial P_{a}}{\partial \phi} \nabla \phi+\frac{\partial P_{a}}{\partial V_{a}} \nabla V_{a}+\sum_{j} \frac{\partial P_{a}}{\partial C_{j}} \nabla C_{j} \\
& =-n_{a} \nabla\left(q_{a} \Phi+m_{a} \phi+\frac{1}{2} m_{a} V_{a}^{2}\right)+\sum_{j} \frac{\partial P_{a}}{\partial C_{j}} \nabla C_{j} .
\end{aligned}
$$

Because the parallel gradient of each $C_{j}$ vanishes, that is, $\Gamma_{a} \cdot \nabla C_{j}=0+\mathcal{O}\left[\left(m_{a} c / q_{a}\right)^{2}\right],(20)$ satisfies parallel equilibrium (11). It then follows from (9) that the perpendicular velocity is given by

$$
\mathrm{V}_{\mathrm{a} \perp}=\frac{c}{n_{\mathrm{a}} q_{\mathrm{a}} \Gamma_{\mathrm{a}}^{2}} \Gamma_{\mathrm{a}} \times \sum_{j} \frac{\partial P_{\mathrm{a}}}{\partial C_{j}} \nabla C_{j} .
$$

This expression for the perpendicular velocity, which is expressed in terms of quantities that are constant along $\Gamma_{a}$, can be used to determine the field-aligned currents in the plasma. It is in a form analogous to the current density perpendicular to the magnetic field in MHD plasma and can be solved for the total velocity in the same manner. The solution is (see HP91 for methodology)

$$
q_{\mathrm{a}} n_{a} \mathrm{~V}_{a}(\mathrm{x})=c \sum_{j} \nabla \mathcal{V}_{a, j} \times \nabla C_{j}
$$

where

$$
\mathcal{V}_{a, j}(\mathbf{x}) \equiv \int_{\Gamma} \frac{\partial P_{a}}{\partial C_{j}} \frac{d \ell}{\Gamma_{a}}
$$

and the label $\Gamma$ on the integral sign means that the integration is taken along $\Gamma_{a}$ from the equator to $x$. This solution is appropriate for a north-south symmetric magnetosphere-ionosphere system such that the 
Birkeland currents at the northern and southern ends of the flux tubes are identical. In general, there is an additional force-free current $\alpha \mathrm{B}$ running from ionosphere to ionosphere. While the results can be derived for more general configurations, including open field lines, we assume a symmetric closed field line situation for simplicity and simultaneously neglect any force-free current. The divergence of (22) is zero and its perpendicular component satisfies (9), showing that (22) is a solution of (5) and (6) in steady state, as required.

The current density is given by the sum of (22) over species:

$$
\mathrm{J}(\mathbf{x})=c \sum_{a} \sum_{j} \nabla \mathcal{V}_{a, j} \times \nabla C_{j} .
$$

The Birkeland current density, that is, the vertical current density at the ionosphere, determined by methods identical to those of HP91, is

$$
J_{r, i}=-c \sum_{a} \sum_{j} \frac{\left|\Gamma_{a r, i}\right|}{\Gamma_{a, e}^{2}} \Gamma_{a, e} \cdot \nabla_{a, e} \mathcal{V}_{a, j} \times \nabla_{a, e} C_{j}
$$

where $\Gamma_{a, e}$ and $\Gamma_{a r, i}$ are evaluated at the equator and ionosphere, respectively, the subscript $r$ means the radial component of a vector, $\nabla_{a, e}$ is a two-dimensional gradient evaluated at the equatorial crossing point of the field line of $\boldsymbol{\Gamma}_{a}$, and $\mathcal{V}_{a, j}$ is evaluated by integration from the equator to the ionosphere. The subscript $e$ denotes the equatorial crossing point of field line of the generalized vorticity and the subscript 0 will denote the equatorial crossing point of a magnetic field line (see Figure 1).

To demonstrate the physical content of (22)-(25), we give an example in terms of Maxwellian plasma. It follows from (17) and (18) that the derivatives of the pressure with respect to the constants are $\partial P_{a} / \partial P_{a, e}=$ $P_{a} / P_{a, e}=\rho_{a} / \rho_{a, e}, \partial P_{a} / \partial \Phi_{e}=q_{a} n_{a}, \partial P_{a} / \partial \phi_{e}=$ $m_{a} n_{a}, \partial P_{a} / \partial \frac{1}{2} V_{a, e}^{2}=m_{a} n_{a}$, and $\partial P_{a} / \partial T_{a, e}=n_{a} q_{a}\left(\Psi_{a}\right.$ $\left.-\Psi_{a, e}\right) / T_{a, e}$. Here we have taken $P_{a, e}$ and $T_{a, e}$ to be independent constants; the equatorial number density is not independent but is related to them by $P_{a, e}=$ $n_{a, e} T_{a, e}$. The charge flux density (22) is

$$
\begin{aligned}
q_{a} n_{a} V_{a}= & c\left(\nabla \int_{\Gamma} \frac{\rho_{a}}{\rho_{a, e}} \frac{d \ell}{\Gamma_{a}} \times \nabla P_{a, e}\right. \\
& +\nabla \int_{\Gamma} \frac{n_{a} d \ell}{\Gamma_{a}} \times \nabla\left(q_{a} \Phi_{e}+m_{a} \phi_{e}+\frac{1}{2} V_{a, e}^{2}\right) \\
& \left.+\nabla \int_{\Gamma} \frac{q_{a} n_{a}\left(\Psi_{a}-\Psi_{a, e}\right)}{T_{a, e}} \frac{d \ell}{\Gamma_{a}} \times \nabla T_{a, e}\right) .
\end{aligned}
$$

If parallel electric fields, gravity, and ion inertia are neglected, this reduces to

$$
q_{a} n_{a} \mathrm{~V}_{a}=c\left(\nabla \int_{B} \frac{d \ell}{B} \times \nabla P_{a, 0}\right.
$$




$$
\left.+\nabla \int_{B} \frac{n_{a} q_{a} d \ell}{B} \times \nabla \Phi_{\circ}\right)
$$

where the label $B$ on the integral sign means that the integration is taken along the magnetic field from the equator, the subscript o refers to the equatorial crossing point of the magnetic field line; we have assumed that the number density is constant along the magnetic field in the absence of a parallel electric field. The perpendicular component is the usual perpendicular velocity, $\mathrm{V}_{\mathrm{a} \perp}=c \mathrm{E} \times \mathrm{B} / B^{2}+\left(c / n_{\mathrm{a}} q_{\mathrm{a}} B^{2}\right) \mathrm{B} \times \nabla P_{\mathrm{a}}$. The current density is given by the sum over species

$$
\mathrm{J}=c\left(\nabla \int_{B} \frac{d \ell}{B} \times \nabla P_{\circ}+\nabla \int_{B} \frac{\eta d \ell}{B} \times \nabla \Phi_{\circ}\right),
$$

where $P_{0}$ is the sum of the electron and ion pressures at the equator; $\eta$ is the charge density and can be assumed to vanish in quasi-neutral plasma. The first term in (28) is Vasyliunas' expression for the total current density due to pressure gradients in isotropic plasma.

Equations (24) and (25) are formally exact, that is, (24) satisfies (1) and (3) exactly (recall, however, that $\left.\Gamma_{a} \cdot \nabla C_{j}=\mathcal{O}\left[\left(m_{a} c / q_{a}\right)^{2}\right]\right)$. However, they are not very useful for analysis or modeling of field-aligned currents. As can be seen by comparison of (26) and (27), the effects of a convection electric field are not only the addition of explicit terms involving the velocity, but changes in the bounce paths as indicated by the differences in integration paths and equatorial values. The difference of the bounce paths, which reflects the effect of the vorticity on the bounce motions of the ions and contains information about the polarization of the plasma, is relatively small. The departure at the ionosphere from the field line occupied by a particle at its equatorial crossing point is of the order of the ratio of the equatorial $E \times B$ drift velocity to the equatorial gyrofrequency, about 10 $\mathrm{km}$ for hydrogen and a $100 \mathrm{~km}$ for oxygen, smaller than desirable for numerical simulations. On the other hand, the smallness of the difference in the bounce paths allows the development of accurate approximations, to which we now turn.

\section{MHD Limit of Two-Fluid Solutions}

\section{Introduction}

The problem of the field-aligned current density is normally approached from the perspective of the MHD momentum equation

$$
\rho \frac{d \mathbf{V}}{d t}+\nabla P+\rho \nabla \phi=\frac{1}{c} \mathrm{~J} \times \mathrm{B}
$$


If inertia and gravity are neglected the solution for the Birkeland current density is [Vasyliunas, 1970]

$$
J_{r, i}=-\frac{c\left|B_{r, i}\right|}{B_{\circ}^{2}} B_{\circ} \cdot \nabla_{\circ} \int \frac{d \ell}{B} \times \nabla_{\circ} P_{\circ}
$$

Here $B_{r, i}$ is the radial component of the magnetic field at the ionosphere, the subscript o means evaluation at the magnetospheric equator, and $\nabla_{0}$ is a two-dimensional gradient in the equatorial plane.

The essential feature that allows the integration along field lines to give (30) is the constancy of the pressure along the magnetic field, $\mathbf{B} \cdot \nabla P=0$. The inclusion of inertia would be an easy matter if there were an analogous simple quantity that was constant along the magnetic field; however, there appears to be no simple field line constant in that case. To see this, note that it follows from (29) in steady state that

$$
\mathrm{B} \cdot \nabla P+\rho \mathrm{B} \cdot \nabla\left(\frac{1}{2} V^{2}+\phi\right)-\rho \mathrm{B} \cdot \mathrm{V} \times(\nabla \times \mathrm{V})=0 .
$$

In steady state MHD approximation, with curl-free electric field related to the bulk velocity by $\mathbf{E}=-\mathbf{V} \times \mathbf{B} / \mathrm{c}$, the third term is $-\rho \nabla \cdot[\mathbf{V} \times(\mathbf{V} \times \mathbf{B})]$, and therefore the parallel equilibrium equation is

$$
\mathrm{B} \cdot \nabla P+\rho \mathrm{B} \cdot \nabla\left(\frac{1}{2} V^{2}-V_{\perp}^{2}+\phi\right)+\rho \nabla \cdot\left(B V_{\|} \mathrm{V}_{\perp}\right)=0
$$

Not only is the pressure not constant along the magnetic field, but there appears to be no simple generalization of the constant pressure relation in terms of other constants. One can show that an equivalent form of the parallel equilibrium condition is

$$
\frac{1}{\rho} \mathrm{B} \cdot \nabla P+\mathrm{B} \cdot \nabla\left(-\frac{1}{2} V^{2}+\phi+\mathrm{V} \cdot \nabla \int V_{\|} d \ell\right)=0 .
$$

if $P=P(\rho)$ along B. The integral of the parallel velocity is evaluated by integration along the magnetic field from the equator to arbitary points along the magnetic field line. The derivation of (33) from (32) is straightforward but tedious. It is done by using the divergence properties of the last term of (32). The only possibly obscure step is to integrate the right-hand side over the surface of a flux tube, use Gauss' law to convert it to a surface integral, substitute the $E \times B$ drift, and perform the surface integral by the methods of HP90. The result is

$$
\int d^{3} x \nabla \cdot\left(B V_{\|} \mathrm{V}_{\perp}\right)=-\oint\left(\int V_{\|} d \ell\right) d \Phi
$$

where $\Phi$ is assumed constant along field lines, the contour integral is taken around any right-handed loop in 
the plasma, and the integral over $d \ell$ is taken from the equatorial plane to the surface defining the ends of the flux tube. Applying Stokes' theorem to the right-hand side of (34) one finds

$$
\int d^{3} x \nabla \cdot\left(B V_{\|} \mathbf{V}_{\perp}\right)=-c \int \nabla \int V_{\|} d \ell \times \nabla \Phi \cdot \mathbf{n} d a
$$

Combining this result, evaluated at the end of the flux tube with $\mathrm{n}=\mathrm{B} / B$, with the results of the more transparent integrals of the remaining terms of (32) gives (33).

\section{MHD Limit}

The direct solution of (29) for the parallel current density presents insurmountable difficulties. Rather than continue with that analysis, we solve the same problem by taking the MHD limit of the two-fluid solutions for the current density. The method is to expand (24) in terms of the particle mass to charge ratios. By retaining only lowest-order terms consistent with the MHD approximation we obtain solutions for the MHD current density. To do this, we reexpress (24) in terms of integrals along the magnetic field lines and constants evaluated at the equatorial crossing points of magnetic field lines rather than field lines of the generalized vorticity, by expanding each to order $\epsilon_{a} \equiv m_{a} c / q_{a}$. The approximation of $C_{j}$ is based on the fact that it is constant along field lines of the generalized vorticity, $\Gamma_{a} \cdot \nabla C_{j}=\mathcal{O}\left(\epsilon_{a}^{2}\right)$. (see discussion after (13)). By expressing $\Gamma_{a}$ in terms of the magnetic field and vorticity, one can write this as the differential equation

$$
\frac{d C_{j}}{d \ell}=-\frac{m_{a} c}{q_{a} B} \omega_{a} \cdot \nabla C_{j}+\mathcal{O}\left(\epsilon_{a}^{2}\right),
$$

where $\ell$ represents arclength along the magnetic field. To order $\epsilon_{a}$ the constant $C_{j}$ appearing on the right-hand side, which refers to the equatorial crossing point of the field lines of the generalized vorticity, can be replaced by $C_{j, 0}$, which refers to the equatorial crossing point of the magnetic field line. Integrating along the magnetic field from the equator one obtains

$$
C_{j} \equiv C_{j, e}=C_{j, \circ}-\frac{m_{a} c}{q_{a}} \int_{B}\left(\omega_{a} \cdot \nabla C_{j, 0}\right) \frac{d \ell}{B}+\mathcal{O}\left(\epsilon_{a}^{2}\right),
$$

(where the label $B$ on the integral sign means that the integral is taken along the magnetic field to arbitrary points in the plasma). The integration constant on the right-hand side is determined by the fact that in the limit that the evaluation point approaches the equator the field lines of the generalized vorticity and magnetic 
field are identical. In a similar manner, one can show that

$$
\begin{aligned}
\mathcal{V}_{a, j} \equiv & \int_{\Gamma} \frac{\partial P_{a}}{\partial C_{j}} \frac{d \ell}{\Gamma_{a}} \\
= & \mathcal{V}_{a, j}^{(0)}-\frac{m_{a} c}{q_{a}} \int_{B}\left(\omega_{a} \cdot \nabla \mathcal{V}_{a, j}^{(0)}\right) \frac{d \ell}{B} \\
& +\mathcal{O}\left(\epsilon_{a}^{2}\right)
\end{aligned}
$$

where

$$
\mathcal{V}_{a, j}^{(0)} \equiv \int_{B} \frac{\partial P_{a}}{\partial C_{j}} \frac{d \ell}{B}
$$

Substituting these results into (24), one finds for the current density $\mathbf{J}(\mathbf{x})=\mathbf{J}^{(0)}(\mathbf{x})+\mathbf{J}^{(1)}(\mathbf{x})$, where

$$
\begin{aligned}
\mathrm{J}^{(0)}(\mathrm{x}) & =c \sum_{j} \sum_{a} \nabla \mathcal{V}_{a, j}^{(0)} \times \nabla C_{j, 0} \\
\mathrm{~J}^{(1)}(\mathbf{x})= & -c \sum_{a} \frac{m_{a} c}{q_{a}} \sum_{j} \\
& \cdot\left[\nabla \mathcal{V}_{a, j}^{(0)} \times \nabla \int_{B}\left(\omega_{a} \cdot \nabla C_{j, 0}\right) \frac{d \ell}{B}\right. \\
& \left.+\nabla \int_{B}\left(\omega_{a} \cdot \nabla \mathcal{V}_{a, j}^{(0)}\right) \frac{d \ell}{B} \times \nabla C_{j, 0}\right] .
\end{aligned}
$$

These hold at arbitrary points, $x$, in the plasma; solutions can be determined, in principle, by constructing the values of the integrals and field line constants as functions of the coordinates by integration from the equator to arbitrary points in the plasma and then taking their local gradients. This is the general form of expansion of the current density and serves as the basis for evaluation of particular cases (i.e., specific distribution functions).

Equations (40) and (41) can be used to determine the Birkeland current density. When evaluated at the ionosphere, each integral appearing on the right-hand side is integrated along magnetic field lines from equator to ionosphere and therefore can be regarded as a function of Euler potentials $\alpha$ and $\beta$ that determine the magnetic field according to $\mathrm{B}=\nabla \alpha \times \nabla \beta$ [see Stern, 1970]. By expressing $J$ in terms of $\alpha$ and $\beta$, one finds for the Birkeland current density

$$
\begin{aligned}
J_{r, i}^{(0)}=-c & \frac{\left|B_{r, i}\right|}{B_{0}^{2}} \mathbf{B}_{\circ} \cdot \sum_{j} \sum_{a} \nabla_{0} \mathcal{V}_{a, j}^{(0)} \times \nabla_{0} C_{j, 0} \\
J_{r, i}^{(1)}= & c \frac{\left|B_{r, i}\right|}{B_{0}^{2}} \mathbf{B}_{\circ} \cdot \sum_{a} \frac{m_{a} c}{q_{a}} \\
& \cdot\left[\nabla_{\circ} \mathcal{V}_{a, j}^{(0)} \times \nabla_{\circ} \int_{B}\left(\omega_{a} \cdot \nabla C_{j, 0}\right) \frac{d \ell}{B}\right. \\
& \left.+\nabla_{\circ} \int_{B}\left(\omega_{a} \cdot \nabla \mathcal{V}_{a, j}^{(0)}\right) \frac{d \ell}{B} \times \nabla_{\circ} C_{j, 0}\right] .
\end{aligned}
$$


Solutions for the Birkeland current density can be determined in much the same manner as the current density in three dimensions, the difference being that the integrals must be evaluated by integration along the magnetic field from the equator to the ionosphere.

\section{Maxwellian Particle Distributions}

Equations (40) and (41) are expressed in completely abstract forms that depend on the constants characterizing the particle distribution functions. To use them for the computation of currents, one must state the distribution functions as functions of the total energy and the constants. Assume, for example, that each equatorial distribution function is a convected isotropic Maxwellian (16). The number density and pressure of each species are to be determined by integration of (12) and (13). To perform the integration, the limits of integration must be considered. Two natural cases are potentials that increase or decrease monotonically from the equator. Of these, we treat only the former. In the latter case the particles see a potential maximum at the equator and are presumably lost in the ionosphere. The monotonically increasing potential is by no means the most general case. Nonmonotonic potentials are possible and the reader should be aware of this restriction on our results. For monotonically increasing effective potential the lower limit of integration is the local (i.e., at the location $\ell$ along the field line) value of the effective potential; the upper limit is $\mathcal{E}=\infty$. The number density and pressure that result from the integration are given by (17) and (18). Substitution for the partial derivatives with respect to the constants (see the text introducing (26)) into (40) leads to the current density

$$
\begin{aligned}
\mathbf{j}^{(0)}= & c \sum_{a}\left(\nabla \int \frac{\rho_{a}}{\rho_{a, 0}} \frac{d \ell}{B} \times \nabla P_{a, 0}\right. \\
& +\nabla \int \frac{n_{a} d \ell}{B} \times \nabla\left(q_{a} \Phi_{\circ}+m_{a} \phi_{\circ}+\frac{1}{2} m_{a} V_{\circ}^{2}\right) \\
& \left.+\nabla \int \frac{q_{a} n_{a}\left(\Psi_{a}-\Psi_{a, 0}\right)}{T_{a, 0}} \frac{d \ell}{B} \times \nabla T_{a, 0}\right) \\
& +\mathcal{O}\left(\epsilon_{+}\right)
\end{aligned}
$$

(cf. (26)). The integrals are along magnetic field lines from the equator to arbitrary points in the plasma; the subscript $B$ on the integral sign will be omitted hereafter. At the same level of approximation the velocity that follows from (22) summed over species is

$$
\rho \mathrm{V}=c \nabla \int \frac{\rho d \ell}{B} \times \nabla \Phi_{\circ}+\mathcal{O}\left(\epsilon_{+}\right)
$$

that is, the dominant contribution has been assumed to be from the electric field gradient, with the pressure, 
gravitational potential, and velocity gradients neglected as being of one higher order in $\epsilon_{+}$. For weak parallel potential drops the perpendicular component is the $E \times$ $B$ drift velocity, $V_{\perp}=V_{E}$. The parallel component of the velocity generally does not vanish [see Schindler and Bim, 1987].

Solutions that satisfy the condition of quasi-neutrality are determined by the condition that the ion and electron number densities be equal, that is, $n=n_{+}=n_{-}$, where \pm denote ions and electrons, respectively. According to (17), this condition is to be satisfied by the solution of

$$
n_{+, e} e^{-e\left(\Psi_{+}-\Psi_{+, e}\right) / T_{+, e}}=n_{-, e} e^{e\left(\Psi_{-}-\Psi_{-, e}\right) / T_{-, e}}
$$

(where $e$ is the charge of the ions, assumed singly ionized) anywhere in the plasma as long the effective potentials of both ions and electrons increase monotonically with distance from the equator. According to (38), the relation between $n_{ \pm, e}$ and $n_{ \pm, 0} \equiv n_{0}$ is

$$
n_{ \pm, e}=n_{\circ} \mp \frac{m_{ \pm} c}{e} \int\left(\omega \cdot \nabla n_{0}\right) \frac{d \ell}{B}+\mathcal{O}\left(\epsilon_{ \pm}^{2}\right),
$$

where $\boldsymbol{\omega}=$ curl $\mathrm{V}$ is to be determined from (45). The condition of quasi-neutrality is then

$$
\begin{aligned}
& \frac{e\left(\Psi_{+}-\Psi_{+, e}\right)}{T_{+, e}}+\frac{e\left(\Psi_{-}-\Psi_{-, e}\right)}{T_{-, e}} \\
& =\ln \left(\frac{n_{+, e}}{n_{-, e}}\right) \\
& =-\frac{m c}{n_{0} e} \int\left(\omega \cdot \nabla n_{0}\right) \frac{d \ell}{B}+\mathcal{O}\left(\epsilon_{+}^{2}\right)
\end{aligned}
$$

where $m=m_{+}+m_{-}$is the sum of the ion and electron masses. The potentials on the left-hand side can be referred to the equatorial crossing point of the magnetic field line by writing

$$
\begin{aligned}
& \frac{e\left(\Psi_{+}-\Psi_{+, \mathrm{o}}\right)}{T_{+, e}}+\frac{e\left(\Psi_{-}-\Psi_{-, o}\right)}{T_{-, e}} \\
& =-\frac{m c}{n_{0} e} \int\left(\omega \cdot \nabla n_{\mathrm{o}}\right) \frac{d \ell}{B} \\
& \quad+\frac{e\left(\Psi_{+, e}-\Psi_{+, \mathrm{o}}\right)}{T_{+, e}}+\frac{e\left(\Psi_{-, e}-\Psi_{-, o}\right)}{T_{-, e}} \\
& \quad+\mathcal{O}\left(\epsilon_{+}^{2}\right) .
\end{aligned}
$$

In the last two terms on the right-hand side we retain only the electrostatic potential, regarding gravity and kinetic energy gradients as one higher order in $\epsilon$. Then, by (38), they can be expressed

$$
\frac{e\left(\Psi_{ \pm, e}-\Psi_{ \pm, \mathrm{o}}\right)}{T_{ \pm, e}}=\mp \frac{m_{ \pm} c}{T_{ \pm, e}} \int\left(\omega \cdot \nabla \Phi_{\circ}\right) \frac{d \ell}{B}+\mathcal{O}\left(\epsilon_{ \pm}^{2}\right) .
$$


By an analysis similar to that outlined in (34) and (35), one can show that

$$
\int\left(\omega \cdot \nabla \Phi_{\circ}\right) \frac{d \ell}{B}=-\frac{1}{c}\left(V^{2}-V_{\circ}^{2}\right)+\frac{1}{c} \mathrm{~V} \cdot \nabla \int V_{\|} d \ell .
$$

The solutions of (49)-(51) for the effective potentials are

$$
\begin{aligned}
\frac{ \pm e\left(\Psi_{ \pm}-\Psi_{ \pm, e}\right)}{T_{ \pm, e}}= & \frac{m}{2 T_{\circ}}\left[-\frac{1}{2}\left(V^{2}-V_{\circ}^{2}\right)+\left(\phi-\phi_{\circ}\right)\right. \\
& \left.+\mathrm{V} \cdot \nabla \int V_{\|} d \ell\right]+\mathcal{O}\left(\epsilon_{+}\right),
\end{aligned}
$$

where $T_{0}$ is the mean value of the electron and ion temperatures at the equator. By (17) and (18) the number density and pressure are

$$
\begin{aligned}
n= & n_{\circ} \exp \left\{\frac { m } { 2 T _ { \circ } } \left[\frac{1}{2}\left(V^{2}-V_{\circ}^{2}\right)-\left(\phi-\phi_{\circ}\right)\right.\right. \\
& \left.\left.-\mathrm{V} \cdot \nabla \int V_{\|} d \ell\right]\right\}+\mathcal{O}\left(\epsilon_{+}\right) \\
P= & P_{\circ} \exp \left\{\frac { m } { 2 T _ { \circ } } \left[\frac{1}{2}\left(V^{2}-V_{\circ}^{2}\right)-\left(\phi-\phi_{0}\right)\right.\right. \\
& \left.\left.-\mathrm{V} \cdot \nabla \int V_{\|} d \ell\right]\right\}+\mathcal{O}\left(\epsilon_{+}\right),
\end{aligned}
$$

valid if $-V^{2} / 2+\phi+\mathrm{V} \cdot \nabla \int V_{\|} d \ell$ increases monontonically with increasing distance from the equator (see (52)). Differentiation of (54) along the magnetic field leads to (33), showing that (53) and (54) satisfy MHD parallel equilibrium (since, by (53) and (54), $P=P(\rho)$ ). The associated electrostatic potential drop is

$$
\begin{aligned}
\frac{e\left(\Phi-\Phi_{\circ}\right)}{T_{-, \circ}}= & -\frac{m_{+}}{2 T_{\circ}}\left[\phi-\phi_{\circ}-\frac{1}{2}\left(V^{2}-V_{\circ}^{2}\right)\right. \\
& \left.-\mathrm{V} \cdot \nabla \int V_{\|} d \ell\right]+\mathcal{O}\left(\epsilon_{+}\right) .
\end{aligned}
$$

(The integral, as usual, is determined by integration from the equator to arbitrary points in the plasma). The entropy per unit mass, which is useful in writing the results, follows from the addition property of the entropy, $\rho s=\sum_{a} n_{a} m_{a} s_{a}$, or

$$
\begin{aligned}
s= & s_{\circ}+\frac{1}{T_{\circ}}\left[-\frac{1}{2}\left(V^{2}-V_{\circ}^{2}\right)\right. \\
& \left.+\left(\phi-\phi_{\circ}\right)+V \cdot \nabla \int V_{\|} d \ell\right]+\mathcal{O}\left(\epsilon_{+}\right),
\end{aligned}
$$

where

$$
s=\frac{3}{2 m} \sum_{a} \ln \left(\frac{P_{a}}{\rho_{a}^{5 / 3}}\right),
$$


and the ions and electrons have each been characterized by three degrees of freedom. Recognizing that the charge density is zero in quasi-neutral plasma, the current density (44) can now be written

$$
\begin{aligned}
\mathrm{J}^{(0)}= & c\left[\nabla \int \frac{\rho}{\rho_{\mathrm{o}}} \frac{d \ell}{B} \times \nabla P_{\mathrm{o}}\right. \\
& +\nabla \int \frac{\rho d \ell}{B} \times \nabla\left(\phi_{\circ}+\frac{1}{2} V_{\circ}^{2}\right) \\
& \left.+\nabla \int \rho\left(s-s_{\mathrm{o}}\right) \frac{d \ell}{B} \times \nabla T_{\mathrm{o}}\right] \\
& +\mathcal{O}\left(\epsilon_{+}\right) .
\end{aligned}
$$

The current density $\mathbf{J}^{(1)}$ can be treated in an analogous manner. We retain only those terms proportional to gradients of the electrostatic potential, regarding those involving thermal properties and gravitation as being of a higher order in $\epsilon_{a}$. Then $J^{(1)}$ is

$$
\begin{aligned}
\mathrm{J}^{(1)}= & -\sum_{a} m_{a} c^{2}\left[\nabla \int \frac{n_{a} d \ell}{B} \times \nabla \int\left(\omega_{a} \cdot \nabla \Phi_{\circ}\right) \frac{d \ell}{B}\right. \\
& \left.+\nabla \int\left(\omega_{a} \cdot \nabla \int \frac{n_{a}\left(\ell^{\prime}\right) d \ell^{\prime}}{B\left(\ell^{\prime}\right)}\right) \frac{d \ell}{B} \times \nabla \Phi_{\circ}\right] \\
& +\mathcal{O}\left(\epsilon_{+}\right) .
\end{aligned}
$$

Since neither the number density nor vorticity depends on the species in MHD approximation, (59) can be summed to yield

$$
\begin{aligned}
\mathbf{J}^{(1)}= & -c^{2}\left[\nabla \int \frac{\rho d \ell}{B} \times \nabla \int\left(\omega \cdot \nabla \Phi_{0}\right) \frac{d \ell}{B}\right. \\
& \left.+\nabla \int(\omega \cdot \nabla M) \frac{d \ell}{B} \times \nabla \Phi_{0}\right]+\mathcal{O}\left(\epsilon_{+}\right),
\end{aligned}
$$

where

$$
M(\mathbf{x}) \equiv \int \frac{\rho d \ell}{B}
$$

is the mass per unit flux, a three-dimensional function of the coordinates determined by integration along magnetic field lines from the equator to $x$. The first term can be written using (51). Using the fact that the vorticity has no divergence, one can rewrite the second term:

$$
\begin{aligned}
\int(\omega \cdot \nabla M) \frac{d \ell}{B}= & \int \nabla \cdot(\mathrm{V} \times \nabla M) \frac{d \ell}{B} \\
= & \int \nabla \cdot\left(\mathrm{V}_{E} \times \nabla_{\|} M+\mathrm{V}_{\|} \times \nabla_{\perp} M\right. \\
& \left.+\mathrm{V}_{E} \times \nabla_{\perp} M\right) \frac{d \ell}{B} .
\end{aligned}
$$

The first term of (62) can be expressed in terms of the electric field and the last can be integrated immediately. 
The result is

$$
\begin{aligned}
\int(\omega \cdot \nabla M) \frac{d \ell}{B}= & -\int \nabla \cdot\left(\frac{\rho c}{B^{2}} \mathbf{E}_{\perp}-V_{\|} \mathbf{e}_{\|} \times \nabla_{\perp} M\right) \frac{d \ell}{B} \\
& +\frac{c}{B^{2}} \mathbf{E}_{\perp} \cdot \nabla M
\end{aligned}
$$

where we have used $\nabla_{\|} M=\rho / B$. In ordinary dielectric media, characterized by the plasma low-frequency dielectric constant $1+4 \pi \rho c^{2} / B^{2}$, the polarization charge density is

$$
\eta_{P}=-\nabla \cdot\left(\frac{\rho c^{2} \mathbf{E}_{\perp}}{B^{2}}\right)
$$

In the present case the change in the number per unit flux on a given magnetic field line due to the presence of an electric field, obtained from (38), is

$$
\begin{aligned}
\delta \int \frac{n_{a} d \ell}{B} & =-\left(\int_{\Gamma} \frac{n_{a} d \ell}{\Gamma_{a}}-\int_{B} \frac{n_{a} d \ell}{B}\right) \\
& =\frac{m_{a} c}{q_{a}} \int\left(\omega_{a} \cdot \nabla \int \frac{n_{a}\left(\ell^{\prime}\right) d \ell^{\prime}}{B\left(\ell^{\prime}\right)}\right) \frac{d \ell}{B} .
\end{aligned}
$$

That is, in comparison with the situation in the absence of an electric field, the particles are displaced from the field line, and have a different flux tube content on the given field line. The change in the charge per unit flux is

$$
\sum_{a} \int \frac{q_{a} \delta n_{a} d \ell}{B}=c \int(\omega \cdot \nabla M) \frac{d \ell}{B}
$$

and represents the polarization charge per unit flux in the presence of an electric field. Consequently, by analogy with ordinary dielectric media, we define the flux tube average of the polarization charge density by

$$
\int \frac{\eta_{P} d \ell}{B} \equiv c \int(\omega \cdot \nabla M) \frac{d \ell}{B}
$$

With these identifications the current density $J^{(1)}$ is

$$
\begin{aligned}
\mathrm{J}^{(1)}= & -c \nabla \int \frac{\rho d \ell}{B} \times \nabla\left(V_{\circ}^{2}-V^{2}+\mathrm{V} \cdot \nabla \int V_{\|} d \ell\right) \\
& -c \nabla \int \frac{\eta_{P} d \ell}{B} \times \nabla \Phi_{\circ}+\mathcal{O}\left(\epsilon_{+}\right) .
\end{aligned}
$$

The total current density is given by the sum of (58) and (68):

$$
\begin{aligned}
\mathrm{J}= & c\left[\nabla \int \frac{\rho}{\rho_{\mathrm{o}}} \frac{d \ell}{B} \times \nabla P_{\circ}\right. \\
& +\nabla \int \frac{\rho d \ell}{B} \times \nabla\left(-\frac{1}{2} V_{\circ}^{2}+\phi_{\circ}\right. \\
& \left.+V^{2}-\mathrm{V} \cdot \nabla \int V_{\|} d \ell\right)
\end{aligned}
$$




$$
\begin{aligned}
& +\nabla \int \rho\left(s-s_{\circ}\right) \frac{d \ell}{B} \times \nabla T_{\circ} \\
& \left.-\nabla \int \frac{\eta_{P} d \ell}{B} \times \nabla \Phi_{\circ}\right]+\mathcal{O}\left(\epsilon_{+}\right) .
\end{aligned}
$$

As previously, solutions can be determined by constructing, by integration from the equator to arbitrary points in the plasma, the integrals and equatorial constants as three-dimensional functions of the coordinates and taking the local gradients. The Birkeland current density, obtained by mapping the radial current density at the ionosphere to equatorial properties as in (42) and $(43)$, is

$$
\begin{aligned}
J_{r, i}= & -c \frac{\left|B_{r, i}\right|}{B_{0}^{2}} B_{\circ} \cdot\left[\nabla_{\circ} \int \frac{\rho}{\rho_{\circ}} \frac{d \ell}{B} \times \nabla_{\circ} P_{\circ}\right. \\
& +\nabla_{\circ} \int \frac{\rho d \ell}{B} \times \nabla_{\circ}\left(-\frac{1}{2} V_{\circ}^{2}+\phi_{\circ}\right. \\
& \left.+V_{i}^{2}-V_{i} \cdot \nabla_{i} \int V_{\|} d \ell\right) \\
& +\nabla_{\circ} \int \rho\left(s-s_{\circ}\right) \frac{d \ell}{B} \times \nabla_{\circ} T_{\circ} \\
& \left.-\nabla_{\circ} \int \frac{\eta_{P} d \ell}{B} \times \nabla_{\circ} \Phi_{\circ}\right]+\mathcal{O}\left(\epsilon_{+}\right)
\end{aligned}
$$

where $V_{i}$ and $\nabla_{i}$ are evaluated at the ionosphere. The nature of the solution is similar to that given by (69) except that the integrals are to be constructed by integration to the ionosphere. If the convection velocity and gravity are neglected, (70) depends only on the pressure gradient and reduces to Vasyliunas' [1970] formula (see (30)). The new information contained is the effects of the convection electric field.

\section{Verification of the Formula}

A necessary condition for (69) and (70) to be a correct description of currents in MHD plasma is that (69) has the same perpendicular current density as that of the MHD momentum equation (29) and simultaneously satisfy current continuity (3). That (69) solves (3) is obvious from the fact that its divergence is zero. In order to help convince the reader that our result is indeed a solution for the currents in MHD plasma, we systematically reduce (69) to (29) (including the effects of the gravitational field). To do this, we write (69) in the form

$$
\begin{aligned}
\mathrm{J}= & c\left(\nabla \int \frac{\rho}{\rho_{\circ}} \frac{d \ell}{B} \times \nabla P_{\circ}+\nabla \int \frac{\rho d \ell}{B} \times \nabla\left(\phi_{\circ}+\frac{1}{2} V_{\circ}^{2}\right)\right. \\
& +\nabla \int \rho\left(s-s_{\circ}\right) \frac{d \ell}{B} \times \nabla T_{\circ} \\
& -c \nabla M \times \nabla \int\left(\omega \cdot \nabla \Phi_{\circ}\right) \frac{d \ell}{B}
\end{aligned}
$$




$$
\left.-c \nabla \int(\omega \cdot \nabla M) \frac{d \ell}{B} \times \nabla \Phi_{\circ}\right)+\mathcal{O}\left(\epsilon_{+}\right),
$$

that is, as the sum of (58) and (60). The perpendicular current density is

$$
\begin{aligned}
\mathbf{J}_{\perp} \equiv & \frac{1}{B^{2}} \mathrm{~B} \times(\mathrm{J} \times \mathrm{B}) \\
= & \frac{c}{B^{2}} \mathrm{~B} \times\left[\frac{\rho}{\rho_{\circ}} \nabla P_{\circ}+\rho \nabla\left(\frac{1}{2} V_{\circ}^{2}+\phi_{\circ}\right)\right. \\
= & +\rho\left(s-s_{\circ}\right) \nabla T_{\circ}-\rho \nabla \int\left(\omega \cdot \nabla \Phi_{\circ}\right) \frac{d \ell}{B} \\
& \left.+c\left(\omega \cdot \nabla \Phi_{\circ}\right) \nabla M-c(\omega \cdot \nabla M) \nabla \Phi_{\circ}\right] \\
& +\mathcal{O}\left(\epsilon_{+}\right),
\end{aligned}
$$

where we have used the facts that $\mathrm{B} \cdot \nabla C_{\circ}=0$ for any field line constant $C_{0}$ and

$$
\mathrm{B} \cdot \nabla \int \frac{F d \ell}{B}=F
$$

for any $F$. Now, it follows from (45) that

$$
\begin{aligned}
\rho \mathrm{V} \times(\nabla \times \mathrm{V})= & -c\left(\omega \cdot \nabla \Phi_{\circ}\right) \nabla M+c(\omega \cdot \nabla M) \nabla \Phi_{\circ} \\
& +\mathcal{O}\left(\epsilon_{+}\right) .
\end{aligned}
$$

Combining (51), (72), and (74), one obtains

$$
\begin{aligned}
\mathrm{J}_{\perp}= & \frac{c}{B^{2}} \mathrm{~B} \times\left[\frac{\rho}{\rho_{\mathrm{o}}} \nabla P_{\circ}\right. \\
& +\rho \nabla\left(V^{2}-\frac{1}{2} V_{\circ}^{2}+\phi_{\circ}-\mathrm{V} \cdot \nabla \int V_{\|} d \ell\right) \\
& \left.+\rho\left(s-s_{\circ}\right) \nabla T_{\circ}-\rho \mathbf{V} \times(\nabla \times \mathrm{V})\right] \\
& +\mathcal{O}\left(\epsilon_{+}\right) .
\end{aligned}
$$

The gradient of the pressure that follows from (54) is

$$
\begin{aligned}
\nabla P= & \frac{\rho}{\rho_{0}} \nabla P_{0} \\
& +\rho \nabla\left[\frac{1}{2}\left(V^{2}-V_{\circ}^{2}\right)-\left(\phi-\phi_{0}\right)-\mathrm{V} \cdot \nabla \int V_{\|} d \ell\right] \\
& +\rho\left(s-s_{0}\right) \nabla T_{0}+\mathcal{O}\left(\epsilon_{+}\right),
\end{aligned}
$$

where we have used $P_{0}=2 n_{0} T_{0}$, the factor of 2 following from the definition of the number density as equal to that of either ions or electrons and the definition of the temperature as the mean value of those of ions and electrons. The perpendicular current density is therefore

$$
\mathrm{J}_{\perp}=\frac{c}{B^{2}} \mathrm{~B} \times\left[\nabla P+\rho \nabla \frac{1}{2} V^{2}\right.
$$




$$
\begin{aligned}
& -\rho \mathrm{V} \times(\nabla \times \mathrm{V})+\rho \nabla \phi]+\mathcal{O}\left(\epsilon_{+}\right) \\
= & \frac{c}{B^{2}} \mathrm{~B} \times[\nabla P+\rho(\mathrm{V} \cdot \nabla) \mathrm{V}+\rho \nabla \phi] \\
& +\mathcal{O}\left(\epsilon_{+}\right),
\end{aligned}
$$

that is, the perpendicular component (69) is just equal to the MHD current density in steady state.

\section{Discussion}

In this paper we have developed a description of Birkeland currents in isotropic MHD plasma to include the effects of ion inertia. The results are valid for timescales long compared to the Alfvén transit time and the particle bounce time. Beginning with a description of the currents in isotropic plasma that is collisionless in its bounce motions, we passed to the MHD limit. The resulting expression, which is specific to isotropic Maxwellian distributions for both ions and electrons, accounts for the currents due to ion inertia as well as those due to pressure gradients in steady state.

The forms of the expressions for the current density can be thought of as generalizations of Vasyliunas' [1970] and Tverskoy's [1982] expressions. They have been integrated along the magnetic field and depend on gradients of magnetospheric plasma in much the same manner. The difference in detail, that derivatives of the local velocity, not just equatorial quantities, appear in (69), appears to be harmless. While this complicates the computation of currents near the equator, Birkeland currents (70) can be computed in the same manner as with Vasyliunas' formula. For a given magnetic field model, for example, Tryganenko [1987] or $R$. Hilmer and G.-H. Voigt (A magnetospheric magnetic field model driven by dynamically varying physical input parameters, submitted to Journal of Geophysical Research, 1994) the different contributions to (69), including the polarization current density, can be mapped to the ionosphere to provide models of fieldaligned currents. The computation is straightforward if it is assumed that the equatorial distribution function and electrostatic potential are known and that the parallel velocity is not important. To a first approximation, regarding the field lines as equipotential, the $E \times B$ drift velocity is then determined along the magnetic field, along with the potential drop and the mass density and pressure.

The expressions for the current density are somewhat more complicated than the similar expressions for the current density in quasi-static plasma [ Vasyliznas, 1970; HP90]. There are four separate contributions to the field-aligned currents: the pressure gradient, the gradient of the kinetic energy per particle, the gravitational 
drift, and convection of polarization charge. The complications arise because equilibrium along the magnetic field is not characterized by constant pressure, as in quasi-static plasma, but depends on the electric field.

The parallel component of the polarization current density (the last term of (69)) represents the diversion of polarization charge. If the polarization current density is defined as

$$
\mathrm{J}^{(P)}=-c \nabla \int \frac{\eta_{P} d \ell}{B} \times \nabla \Phi_{\circ}
$$

then the parallel current density is

$$
J_{\|}^{(P)}=c \frac{\mathrm{B} \times \nabla \Phi_{\circ}}{B} \cdot \nabla \int \frac{\eta_{P} d \ell}{B}=B \mathrm{~V}_{E} \cdot \nabla \int \frac{\eta_{P} d \ell}{B},
$$

and is equal to the time rate of change of the polarization charge density. The origin of the Birkeland current density associated with the polarization is the neutralization of polarization charge by electrons. The convection electric field has two simultaneous effects in this connection: it polarizes the plasma by displacing the bounce motions of ions from electrons and it convects both ions and electrons at the $E \times B$ drift velocity. Changes in the convection electric field or the density along the drift path change the polarization charge in a given flux tube. To maintain quasi-neutrality, electrons must move to neutralize the total charge. They cannot accomplish the neutralization by the $E \times B$ drift, which is in a direction perpendicular to the polarization displacement. Instead, they must neutralize the excess charge by closing through the ionosphere.

The effects of polarization are normally discussed in terms of the parallel component of the ion vorticity, $\omega_{\|}=\mathrm{B} \cdot \omega / B$. In general, there are separate contributions due to the parallel and perpendicular components of the vorticity. The contribution of polarization to the Birkeland current density written in terms of the parallel and perpendicular components of the vorticity is

$$
J_{\|}^{(P)}=-B V_{E} \cdot \nabla \int\left(\frac{\rho \omega_{\|}}{B}+\omega_{\perp} \cdot \nabla M\right) \frac{d \ell}{B},
$$

The contribution of the parallel vorticity is familiar, albeit in slightly different form, from the work of Hasegawa and Sato [1979]. Physically, it corresponds to the change in the volume occupied by ions in a given magnetic flux tube due to the presence of an electric field. The second term, explicitly neglected in their analysis, describes the effects of the perpendicular vorticity and describes the effects of the displacement of bounce paths of the ions from those of electrons.

The present results provide a direct way of calculating within a numerical model sources of current due to gradients of the velocity which can reasonably be expected 
to make significant contributions in boundary layers and near the inner edge of the plasma sheet. The application of the resulting expressions may be useful for the analysis of Birkeland currents that originate in the low-latitude boundary layer, the plasma sheet boundary layer, and possibly the inner edge region of the plasma sheet. In the low-latitude boundary layer the solar wind velocity brakes from a few hundred kilometers per second tailward to a few kilometers per second earthward with flow speeds of the order of the ion thermal speed. Quite aside from the effects of viscosity, the velocity shears are likely to generate significant Birkeland currents. In the plasma sheet boundary layer, parallel flows of the order of the ion thermal speed are observed on closed field lines and, according to Schindler and Birn [1987], have a large gradient near the separatrix between open and close field lines. Reference to (70) and (62) suggests that this region is a source of field-aligned current. A further possible source is near the inner edge of the plasma sheet. Westward ion drift paths from the tail adjoin eastward drift paths that circulate the Earth [see Erickson et al., 1991]. Across this layer the electric field reverses and the density need not be continuous, making it a candidate for Birkeland currents that originate in the polarization of the plasma.

Acknowledgments. The Editor thanks two referees for their assistance in evaluating this paper. The authors gratefully acknowledge the support and encouragement of Bill Burke, useful conversations with Dick Wolf, and the assistance of the referees. This work was supported by Air Force Office of Scientific Research Task 2311G5, NASA SR\&T under grants NAGW-2627, NAGW-2856, and NAG 5-1573, and USAF contract number F19628-90-K-0003. 


\section{References}

Akasofu, S.-I., The magnetospheric currents: An introduction, in Magnetospheric Currents, edited by T. A. Potemra, p. 29, AGU, Washington, D. C., 1984.

Birn, J., and M. Hesse, The substorm current wedge and field-aligned currents in MHD simulations of magnetotail reconnection, J. Geophys. Res., 96, 1611, 1991.

Büchner, J., and L. M. Zelenyi, Regular and chaotic charged particle motions in magnetotaillike field reversals, 1, Basic theory of trapped motion, J. Geophys. Res., 94, 11,821, 1989.

Chiu, Y. T., and D. J. Gorney, Eddy intrusion of hot plasma into the polar cap and formation of polar-cap arcs, Geophys. Res. Lett, 10, 463, 1983.

Coley, W. R., Spatial relationship of field-aligned currents, electron precipitation, and plasma convection in the auroral oval, J. Geophys. Res., 88, 7131, 1983.

Eastman, T. E., and E. W. Hones, Characteristics of the magnetospheric boundary layer and magnetopause layer as observed by Imp 6, J. Geophys. Res., 84, 2019, 1979.

Eastman, T. E., E. W. Hones, S. J. Bame, and J. R. Asbridge, The magnetospheric boundary layer: Site of plasma, momentum and energy transfer from the magnetosheath into the magnetosphere, Geophys. Res. Lett., $3,685,1976$.

Eastman, T. E., L. A. Frank, W. K. Peterson, and W. Lennartsson, The plasma sheet boundary layer, $J$. Geophys. Res., 89, 1553, 1984.

Eastman, T. E., L. A. Frank, and C. Y. Huang, The boundary layers as the primary transport regions of the earth's magnetotail, J. Geophys. Res., 90, 9541, 1985.

Erickson, G. M., R. W. Spiro, and R. A. Wolf, The physics of the Harang discontinuity, J. Geophys. Res., 96, 1633, 1991.

Galperin, Yu. I., and A. V. Volosevich, The ARCAD-3 project and the theory of auroral structures, Can J. Phys., $67,719,1989$.

Hasegawa, A., and T. Sato, Generation of field aligned current during substorm, in Dynamics of the Magnetosphene, edited by S.-I. Akasofu, p. 529, D. Reidel, Norwell, Mass., 1979.

Heinemann, M., and D. H. Pontius, Jr., Representations of currents and magnetic fields in isotropic magnetohydrostatic plasma, J. Geophys. Res., 95, 251, 1990.

Heinemann, M., and D. H. Pontius, Jr., Representations of currents and magnetic fields in anisotropic magnetohydrostatic plasma, 2, General theory and examples, $J$. Geophys. Res., 96, 17,609, 1991.

Hines, C. O., The energization of plasma in the magnetosphere: Hydromagnetic and particle-drift appoaches, Planet. Space Sci., 10, 239, 1963.

Hones, E. W., Jr., S. J. Bame, and J. R. Asbridge, Proton flow measurements in the magnetotail plasma sheet made with Imp 6, J. Geophys. Res., 81, 227, 1976.

Kennel, C. F., and H. E. Petschek, Limit on stably trapped particle fluxes, J. Geophys. Res., 71, 1, 1966.

Lyons, L. R., Formation of discrete auroral current and potentials, J. Geophys. Res., 86, 1, 1981.

Macmahon, A., Finite gyro-radius corrections to the hydromagnetic equations for a Vlasov plasma, Phys. Fluids, 8, 
$1840,1965$.

McFadden, J. P., C. W. Carlson, and M. H. Boehm, Fieldaligned electron precipitation at the edge of an arc, $J$. Geophys. Res., 91, 1723, 1986.

Northrop, T. G., Adiabatic Motion of Charged Particles, Interscience, New York, 1963.

Nötzel, A., K. Schindler, and J. Birn, On the cause of approximate pressure isotropy in the quiet near-Earth plasma sheet, J. Geophys. Res., 91, 8293, 1985.

Ogino, T., A three-dimensional MHD simulation of the interaction of the solar wind with the Earth's magnetosphere: The generation of field aligned currents, $J$. Geophys. Res., 91, 6791, 1986.

Phan, T. D., B. U. Ö. Sonnerup, and W. K. Lotko, Selfconsistent model of the low-latitude boundary layer, $J$. Geophys. Res., 94, 1281, 1989.

Rossi, B., and S. Olbert, Introduction to the Physics of Space, McGraw-Hill, New York, 1970.

Rostoker, G., and R. Boström, A mechanism for driving the gross Birkeland current configuration in the auroral oval, J. Geophys, Res., 81, 235, 1976.

Schindler, K., and J. Birn, On the generation of field-aligned plasma flow at the boundary of the plasma sheet, J. Geophys. Res., 92, 95, 1987.

Siscoe, G., and J. Fedder, Report on 1993 Snowmass Workshop, GEM Mess., 3 (12), 1993.

Sonnerup, B. U. Ö., Theory of the low-latitude boundary layer, J. Geophys. Res., 85, 2017, 1980.

Southwood, D. J., and M. G. Kivelson, An approximate description of field-aligned currents in a planetary magnetosphere, J. Geophys. Res., 96, 67, 1991.

Stasiewicz, K., A fluid finite ion Larmor radius model of the magnetopause boundary layer, J. Geophys. Res., 94, 8827, 1989.

Stasiewicz, K., A global model of gyroviscous field line merging at the magnetopause, J. Geophys. Res., 96, 77, 1991.

Stern, D. P., Euler potentials, Am. J. Phys., 38, 494, 1970.

Stiles, G. S., E. W. Hones, Jr., S. J. Bame, and J. R. As bridge, Plasma sheet pressure anisotropies, J. Geophys. Res., 83, 3166, 1978.

Tsyganenko, N. A., Global quantitative models of the geomagnetic field in the cislunar magnetosphere for different disturbance levels, Planet. Space Sci., 35, 1347, 1987.

Tverskoy, B. A., Longitudinal currents in the magnetosphere, Geomagn. Aeron., 22, 812, 1982.

Vasyliunas, V. M., Mathematical models of magnetospheric convection and its coupling to the ionosphere, in Particles and Fields in the Magnetosphere, edited by B. M. McCormac, p. 60, D. Reidel, Norwell, Mass., 1970.

G. M. Erickson, Center for Space Physics, Boston University, Boston, MA 02215. (e-mail: Internet.erickson@buasta. bu.edu)

M. Heinemann, Phillips Laboratory, Hanscom Air Force Base, MA 01731-3010. (e-mail: SPAN.afgl::heinemann)

D. H. Pontius Jr., Bartol Research Institute, Newark, DE 19716. (e-mail: Internet.pontius@bartol.udel.edu)

(Received July 6, 1992; November 29, 1993; accepted January 12, 1994.) 
HEINEMANN ET AL.: INERTIAL CURRENTS IN ISOTROPIC PLASMA HEINEMANN ET AL.: INERTIAL CURRENTS IN ISOTROPIC PLASMA HEINEMANN ET AL.: INERTIAL CURRENTS IN ISOTROPIC PLASMA HEINEMANN ET AL.: INERTIAL CURRENTS IN ISOTROPIC PLASMA HEINEMANN ET AL.: INERTIAL CURRENTS IN ISOTROPIC PLASMA HEINEMANN ET AL.: INERTIAL CURRENTS IN ISOTROPIC PLASMA HEINEMANN ETT AL.: INERTIAL CURRENTS IN ISOTROPIC PLASMA HEINEMANN ET AL.: INERTIAL CURRENTS IN ISOTROPIC PLASMA HEINEMANN ET AL.: INERTIAL CURRENTS IN ISOTROPIC PLASMA HEINEMANN ET AL.: INERTIAL CURRENTS IN ISOTROPIC PLASMA HEINEMANN ET AL.: INERTIAL CURRENTS IN ISOTROPIC PLASMA HEINEMANN ET AL.: INERTIAL CURRENTS IN ISOTROPIC PLASMA HEINEMANN ET AL.: INERTIAL CURRENTS IN ISOTROPIC PLASMA HEINEMANN ET AL.: INERTIAL CURRENTS IN ISOTROPIC PLASMA HEINEMANN ET AL.: INERTIAL CURRENTS IN ISOTROPIC PLASMA HEINEMANN ET AL.: INERTIAL CURRENTS IN ISOTROPIC PLASMA HEINEMANN ET AL.: INERTIAL CURRENTS IN ISOTROPIC PLASMA HEINEMANN ET AL.: INERTIAL CURRENTS IN ISOTROPIC PLASMA HEINEMANN ET AL.: INERTIAL CURRENTS IN ISOTROPIC PLASMA HEINEMANN ET AL.: INERTIAL CURRENTS IN ISOTROPIC PLASMA HEINEMANN ET AL.: INERTIAL CURRENTS IN ISOTROPIC PLASMA HEINEMANN ET AL.: INERTIAL CURRENTS IN ISOTROPIC PLASMA HEINEMANN ET AL.: INERTIAL CURRENTS IN ISOTROPIC PLASMA HEINEMANN ET AL.: INERTIAL CURRENTS IN ISOTROPIC PLASMA HEINEMANN ET AL.: INERTIAL CURRENTS IN ISOTROPIC PLASMA HEINEMANN ET AL.: INERTIAL CURRENTS IN ISOTROPIC PLASMA 
Figure 1. Schematic illustration of the relation between ion bounce paths and the magnetic field. The perpendicular electric field points outward, as indicated. Ions are displaced from the magnetic field in the direction of the perpendicular electric field and follow a bounce trajectory indicated by the dashed line. Electrons, because of their small mass, essentially follow the magnetic field, indicated by the solid line. The equatorial crossing point of the ion bounce path is indicated by $e$ and that of the magnetic field by 0 . 


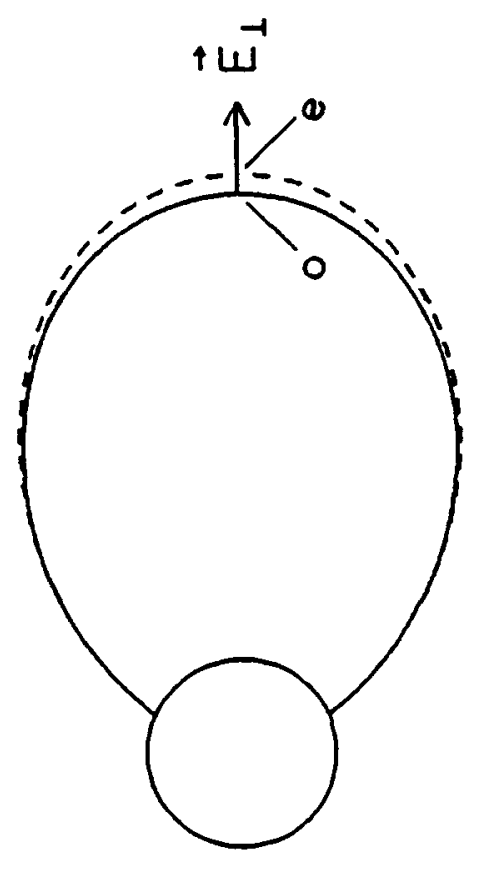

